Internist 2011 · 52:493-494

DOI 10.1007/s00108-011-2846-z

Online publiziert: 20. April 2011

(c) Springer-Verlag 2011

\author{
H. Haller ${ }^{1}$ - B.E. Strauer ${ }^{2}$ \\ ${ }^{1}$ Klinik für Nieren- und Hochdruckerkrankungen, Medizinische Hochschule Hannover \\ ${ }^{2}$ Heinrich-Heine-Universität, Düsseldorf
}

\title{
Endorganschäden bei Diabetes
}

Endorganschäden sind die Ursache der gesteigerten kardiovaskulären Morbidität und Mortalität bei Patienten mit Diabetes mellitus. Die Schäden treten bevorzugt an einer kleinen Zahl von Organen auf, wie Niere, Herz, Milz und Gehirn. Diese Organe zeigen Besonderheiten ihrer Organdurchblutung, da sie von Arterien versorgt werden, die im Anschluss an ihre nutritiven Versorgungsstrukturen keine nachgeschalteten Arterien, keinen zwischengeschalteten Umgehungskreislauf und keine Kollateralen aufweisen. Dies bedeutet, dass die gesamte Organdurchblutung von der Intaktheit dieser Endarterien abhängt. Diese Arterien haben somit den physiologischen Versorgungsauftrag, die von ihnen durchbluteten Organe ausreichend mit Blut, Sauerstoff und Substraten zu versorgen. Dies schließt nicht aus, dass sich Kollateralen und Umgehungskreisläufe ausbilden können, wie z. B. am Herzen, allerdings gehören diese Zusatzkreisläufe primär nicht zur Definition der Endorgandurchblutung.

Die Durchblutung der Endorgane wird durch eine Reihe von Einflussfaktoren beeinflusst: Arterielle Hypertonie, Diabetes mellitus, rheologische Faktoren, Hyperlipoproteinämien, Entzündungsprozesse und Sepsis sind die wichtigsten Risikofaktoren. Bei Störungen der Endorganzirkulation, z. B. durch arteriosklerotischen Verschluss oder eine thrombotische Verlegung der Endarterie, kommt es zum anämischen bzw. ischämischen Infarkt. Zwar sind auch die Äste der Pulmonalarterie und der Pfortader Endarterien, es gibt allerdings neben diesen Gefäßen in Lunge und Leber auch andere, selbstständige arterielle Gefäße, die dem Organ nach Verschluss noch Blut in meist ausreichender Menge zuführen können. Somit kann es hier zu hämorrhagischen Infarkten kommen.

\section{$>$ Endorgane sind in besonders hohem Maße abhängig von Veränderungen der Gefäßstruktur}

Die von der Endstrombahn (Arterien, Arteriolen) versorgten Organe (Endorgane) sind in besonders hohem Maße abhängig von Veränderungen der Gefäßstruktur einschließlich der arteriellen Wandveränderungen, vorhandener Kollateralen und vom Gefäßinhalt, d. h. den Fließeigenschaften des die Endarterien durchströmenden Blutes. Somit spielen gefäßstrukturelle Faktoren einerseits und rheologische Faktoren andererseits eine durchblutungslimitierende Rolle. Risikofaktoren sind insbesondere die Volkskrankheiten arterielle Hypertonie und Diabetes mellitus.

Der Hochdruck betrifft v. a. die Gefäßwandstruktur: Als Folge der chronischen Druckbelastung kommt es zur Hypertrophie aller 3 Wandschichten (Intima, Media, Adventitia), präferenziell der Media. Dadurch nimmt die Wanddicke-Radius-Relation der Arteriolen zu, am Herzen steigt der Koronarwiderstand an, und die Koronarreserve nimmt ab. Die Ischämiebereitschaft des Organs ist gesteigert.
Bei Gefäßentzündungen (Vaskulitis) sind ebenfalls Wandstrukturveränderungen vorhanden.

Beim Diabetes mellitus sind sowohl Wandstrukturveränderungen als auch rheologische Störungen zu nennen. Bei Hyperlipoproteinämien kommen überwiegend rheologisch bedingte Einflüsse, die eine Perfusionsstörung auslösen können, in Betracht, desgleichen bei der Summe der Hyperviskositätssyndrome, bei denen der rheologische Faktor weit im Vordergrund stehen kann. Oft sind kombinierte Formen zwischen Gefäßwandstrukturveränderungen, rheologischen Einflüssen und Diffusionsstörungen, wie bei Hyperlipoproteinämien, vorhanden. In letzter Zeit werden auch Störungen in der Kapillarstrombahn mit Untergang der Kapillaren und Rarefizierung der Endstrombahn als Ursache der Endorganschädigung diskutiert.

Die vorliegende Ausgabe von Der Internist hat das Ziel, die klinisch besonders wichtigen Krankheitsbilder der Endorganschäden $\mathrm{zu}$ fokussieren, da sie oft bei sog. Volkskrankheiten auftreten (arterielle Hypertonie, Diabetes mellitus, Hyperlipoproteinämien $u$. a.) und besonders wichtige Organe betreffen (Herz, Gehirn, Nieren u. a.). Neben der Darstellung der pathophysiologischen und diagnostischen Zusammenhänge ist es das Ziel, die Bedeutung der Endorganschäden nicht nur in Hinblick auf die eingetretene und oft nicht mehr reversible Läsion darzustellen, sondern darüber hinaus auch die Krankheitsursachen, vorrangig aber die beein- 
flussenden Faktoren und die therapeutischen Möglichkeiten im Sinne der Therapie der internistischen Grundkrankheit zu beschreiben.

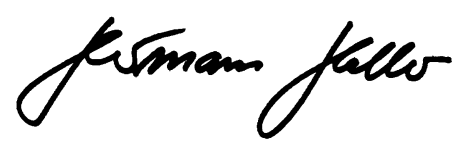

H. Haller

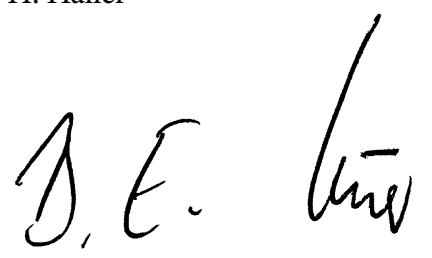

B.E. Strauer

\section{Korrespondenzadresse}

\section{Prof. Dr. H. Haller}

Klinik für Nieren- und Hochdruckerkrankungen,

Medizinische Hochschule Hannover

Carl-Neuberg-Straße 1, 30625 Hannover

Haller.Hermann@mh-hannover.de

\section{Literatur}

1. Emerging Risk Factors Collaboration, Seshasai SR, Kaptoge S, Thompson A (2011) Diabetes mellitus, fasting glucose, and risk of cause-specific death. $\mathrm{N}$ Engl J Med 364:829-841

2. Schmieder R (2010) Hypertoniebedingte Endorganschäden. Dtsch Ärztebl 107:866-873

3. Strauer BE, Schuster HP (2003) Endorganschäden. Internist 44:785

\section{Ideenwettbewerb „Ist Rheuma heilbar?"}

Gesucht ist die beste Idee für einen neuen „Weg zur Heilung" von heute noch unheilbaren rheumatischen Erkrankungen. Gefragt sind Wissenschaftler, Ärzte, junge Forscher und Forschergruppen, die ihre beste Idee zur Entwicklung eines Heilverfahrens als wissenschaftliches Konzept allgemeinverständlich formulieren und bei der Rheumastiftung einreichen können. Die beste Idee wird mit 2.500 Euro ausgezeichnet.

Die Rheuma Stiftung wird sich für die Förderung realistischer Projekte einsetzen.

Beschreiben Sie Ihr wissenschaftliches Konzept allgemeinverständlich auf maximal 5 Seiten und schicken Sie es an: info@ rheumastiftung.org

Einsendeschluss ist der 31. Juli 2011

Quelle: Rheumastiftung, www.rheumastiftung.org

\section{Deutsches Zentrum für Diabetesforschung eröffnet}

Mit dem Ende 2010 eröffneten "Deutschen Zentrum für Diabetesforschung" (DZD) sollen neue Perspektiven für Prävention, Therapie und Diagnose von Diabetes geschaffen werden.

Offene Fragen der Diabetesforschung sollen durch einen integrativer Forschungsansatz geklärt werden. Dafür sieht das DZD ein verstärktes Engagement in der Grundlagenforschung, in klinischen und translationalen Studien, bei sozioökonomischen Fragestellungen und in der Verbesserung von Prävention, Therapie und Patientenversorgung vor.

Nach dem Deutschen Zentrum für Neurodegenerative Erkrankungen ist das DZD das zweite Deutsche Zentrum für Gesundheitsforschung. Vier weitere Zentren zur Bekämpfung wichtiger Volkskrankheiten auf den Gebieten Infektion, Lungenerkrankungen, Herz-Kreislauferkrankungen und Krebs werden 2011 gegründet.

Das aus gleichberechtigten Verbundpartnern bestehende Zentrum führt bundesweit die besten Wissenschaftlerinnen und Wissenschaftler aus Grundlagen-, Versorgungs- und translationaler Forschung zusammen. Verbundpartner des DZD sind: Das Deutsche Diabetes-Zentrum Düsseldorf (DDZ), das Deutsche Institut für Ernährungsforschung Potsdam-Rehbrücke (DIfE), das Helmholtz Zentrum München - Deutsches Forschungszentrum für Gesundheit und Umwelt, sowie die PaulLangerhans-Institute der Universitäten Dresden und Tübingen.

Erklärtes Ziel des DZD ist es, Lücken in der Forschungskette zu schließen und den Weg von der Grundlagenforschung zur tatsächlichen Anwendung beim Patienten zu verkürzen.

Quelle: Deutsches Zentrum für Diabetesforschung, www.dzd-ev.de 